\title{
POESÍA Y CUIDADOS: UN INSTRUMENTO PARA LA GESTIÓN DE EMOCIONES Y SENTIMIENTOS EN ENFERMERÍA
}

POETRY AND CARE: AN INSTRUMENT TO MANAGE EMOTIONS AND FEELINGS IN NURSING

POESIA E CUIDADOS: UM INSTRUMENTO PARA O GERENCIAMENTO DE EMOÇÕES E SENTIMENTOS EM ENFERMAGEM

José Siles-González

Universidad de Alicante. España.

jose.siles@ua.es

ORCID: 0000-0003-3046-639X

María del Carmen Solano-Ruiz.

Universidad de Alicante. España.

ORCID: 0000-0001-8720-8397
DOI: https://doi.org/10.22235/ech.v6i2.1463

Recibido: 24/09/2017

Aceptado: 01/11/2017

\section{RESUMEN}

El objetivo de este estudio consiste en desarrollar una herramienta de reflexión emocional y de sentimientos que contribuyan a la gestión emocional y de los sentimientos de los alumnos de enfermería en sus prácticas clínicas. El método empleado ha consistido en utilizar un grupo de poemas derivados de experiencias clínicas como base para la identificación y análisis de emociones y sentimientos. Asimismo, se emplearon las orientaciones de Vigotsky y Heller para facilitar el análisis de las emociones y los sentimientos. Más allá de la concienciación de los futuros profesionales de la importancia de centrar el cuidado en el paciente, el gran reto consiste en dotar a los enfermeros de la capacidad cognitiva y las destrezas emocionales necesarias para gestionar el aluvión de sentimientos complejos e intensos que emergen durante la aplicación del proceso de enfermería con el fin de prepararlos para el trabajo emocional inherente a su trabajo y disminuir el riesgo de "burnout". Se concluye que los poemas inspirados en experiencias clínicas constituyen una herramienta pertinente para facilitar el trabajo emocional y de sentimientos generados durante las prácticas clínicas.

Palabras clave: Enfermería, Poesía, Proceso de Enfermería, Enfermería Holística, Investigación Metodológica en Enfermería.

\section{ABSTRACT:}

The aim of this study is to develop a tool to think about emotions and feelings, in order to facilitate their management in nursing students in their clinical practices. The method used has been based on the orientations of Vigotsky and Heller to facilitate the classification and analysis of emotions and feelings. Likewise, a selection of poems derived from clinical experiences was used as a basis for the identification and analysis of emotions and feelings. Beyond the awareness of future professionals of the importance of focusing care practices on the patient, the great challenge is to provide nurses with the cognitive capacity and emotional skills necessary to manage the flood of complex and intense feelings that emerge during the application of the nursing process, in order to prepare them for the flow of emotions arising from their work and to decrease the risk of burnout. In conclusion, poems inspired by clinical experiences constitute a relevant tool to facilitate emotional work and feelings generated during clinical practices. The poetry of care contributes to the understanding and emotional regulation and it is a way of channeling the feelings that emerge in the students during their clinical practices.

Keywords: Nursing, Poetry, Nursing Process, Holistic Nursing, Nursing Methodology Research. 


\section{RESUMO:}

O objetivo deste estudo consiste em uma ferramenta de reflexão sobre as emoções e os sentimentos para facilitar a gestão emocional e de sentimentos dos alunos de enfermagem em sus práticas clínicas. O método empregado com base nas orientações de Vygotsky e Heller para facilitar a classificação e análise das emoções e os sentimentos. Além disso, utilizou-se uma escolha de poemas de saídas clínicas como base para a identificação e análise de emoções e sentimentos. Mais além da conceituação dos futuros profissionais da importância do atendimento no paciente, o gran reto consiste em dotar os enfermeiros da capacidade cognitiva e as emoções necessárias para gerenciar o aluvião de sentimentos complexos e intensos que emergem durante a aplicação do processo de enfermagem com o fim de preparação para o fluxo emocional inerente a seu trabalho e diminuir o risco de "queimar". Os poemas inspirados em experiências clínicas constituem uma ferramenta pertinente para facilitar o trabalho emocional e de sentimentos generados durante as práticas clínicas. A poesia de cuidados com a consciência e a regulação emocional e a constituição de canalizar os sentimentos que emergem nos alunos durante as práticas clínicas.

Palavras-chave: Enfermería, Poesia, Processo de Enfermagem, Enfermagem Holística, Pesquisa Metodológica em Enfermagem.

\section{INTRODUCCIÓN}

El problema de este trabajo (y su justificación) se cimenta en el hecho incontrovertible de que la acción de enfermería implica un contacto entre personas (cuidador-cuidado) que facilita la generación de emociones y sentimientos muy variados en cuanto a tipología e intensidad. Las emociones son reacciones a situaciones que se pueden dar durante la práctica clínica y constituyen formas de adaptación a las contingencias ambientales y personales que son características del trabajo enfermero. Las emociones, por tanto, no evolucionan como sentimientos conscientes sino como especializaciones de la conducta y adaptaciones fisiológicas tal como afirman Darwin y Vigotsky, que según Sartre se vinculan con la fenomenología, y que tienen una repercusión en el rendimiento de los alumnos y profesionales de enfermería (1-4).

Los principales actos de expresión de hombres y animales no son aprendidos sino que son innatos o heredados. Las emociones son universales y se expresan de forma no verbal (son las mismas independientemente de las variaciones culturales y geográficas). Tanto las emociones como los sentimientos deben valorarse para considerar su incidencia en la alteración de la atención, la motivación e incluso el estrés, por lo que tienen una gran importancia en el rendimiento del alumno y/o el profesional. Mientras las emociones tienen una rápida respuesta conductual, dado que funcionan como catalizadores del entorno propiciando impulsos favorables o desfavorables hacia ciertas situaciones, personas, acciones, ideas, etcétera, los sentimientos se construyen como consecuencia de la evolución de una emoción (o grupo asociado de ellas), son más estables y forman parte de la conciencia de la persona.

En consecuencia, en este estudio se ha partido del objetivo general de desarrollar una herramienta de reflexión sobre las emociones y los sentimientos que contribuya a la gestión emocional y de los sentimientos de los alumnos de enfermería en sus prácticas clínicas. Asimismo, se ha pretendido alcanzar los siguientes objetivos específicos:

- Identificar las emociones y los sentimientos inspirados por poemas relacionados con la práctica clínica.

- Valorar la incidencia de las emociones y sentimientos en la práctica clínica de los profesionales de enfermería.

\section{Cuestiones de investigación:}

- ¿ ¿Puede la poesía contribuir a la reflexión y gestión de emociones y sentimientos de alumnos durante sus prácticas clínicas?

- ¿ ¿Qué tipo de emociones y sentimientos experimentan los profesionales de enfermería durante sus actuación profesional?

Para contextualizar la temática de este estudio se han revisado aportaciones de diferentes autores que se centran en aspectos relevantes del mismo. Betancourt estudió el impacto de las prácticas clínicas en los docentes desde una perspectiva fenomenológica en la que las vivencias están muy vinculadas a los sentimientos y las emociones (3). En esta misma línea, Siles y Solano han valorado la incidencia de la labor tutorial de los docentes durante el período de prácticas clínicas (5). Una investigación centrada en este mismo tema describe los sentimientos y las emociones que presentan los estudiantes de enfermería de la Universidad Pontificia Bolivariana (UPB) durante el desarrollo de las prácticas clínicas y emplean la teoría de los sentimientos de Agnes Heller para el análisis y ordenación de sus datos (6). Otros estudios han analizado la estructura de los cuidados identificando tres componentes esenciales: organización, trabajo físico y, finalmente, labor emocional (7). Con esta visión en la que el trabajo enfermero está indisolublemente ligado al trabajo emocional y a los sentimientos se han desarrollado otros estudios como los de López y Siles y Solano (811). Todos ellos, de una $u$ otra forma, se centran en las ideas de Harre, quien describe y explica la construcción social de los sentimientos reflejando la idoneidad del trabajo enfermero (12-16). Este carácter estructural que constata la omnipresencia de las dimensiones emocionales en la interacción enfermera-paciente, se refleja también en la perspectiva de los propios pacientes quienes sostienen la idea de que los buenos enfermeros, por encima de los aspectos técnicos, deben desarrollar competencias emocionales y de sentimientos (15). Asimismo, otros estudios han analizado las características del "proceso de enfermería" en relación al fenómeno de las emociones y los sentimientos $(14,17)$.

Respecto a la relación entre poesía y enfermería se han utilizado diversas aportaciones que han contribuido a fundamentar e interpretar los resultados de este estudio sobre la poesía de los cuidados constatando su importancia para la profesión (9, 
$10,19,20)$. Para hablar de la poesía de los cuidados es necesario, previamente, clarificar sus relaciones con dos conceptos que inciden en su producción: arte y estética en el contexto de los cuidados. La enfermería es un arte que, a través de la interacción entre dos personas (paciente y profesional), genera una obra artística en cuya producción participan: conocimiento, técnica, intuición y sensibilidad $(9,10,11)$. La herramienta básica de trabajo en cuidados es el proceso de enfermería, que tiene sus orígenes durante la década de los sesenta; ha sido descrito como una teoría filosófica (o tendencia ideológica en enfermería) y como un método de trabajo. Desde la perspectiva filosófica o ideológica el proceso de enfermería ha promovido el cuidado centrado en el paciente en vez del cuidado centrado en tareas $(14,17)$. El proceso de enfermería, por tanto, debería contribuir mediante la interacción cuidador-cuidado al establecimiento de una dinámica relacional que facilita la construcción social de las emociones y los sentimientos (12).

\section{Metodología}

Se ha partido de los presupuestos del paradigma sociocrítico de Habermas, dado que sus principios potencian la participación de los discentes en su proceso educativo y el rol del profesional de enfermería es, fundamentalmente, un agente de cambio y comunicación facilitador, a su vez, de la participación de los pacientes en el proceso de enfermería (21).

En este trabajo se dota a los alumnos de una herramienta la poesía- para ayudarles a expresar sus sentimientos y emociones mediante un taller pedagógico (taller de poesía de los cuidados en el que se les facilita un conjunto de poemas inspirados en la práctica clínica de enfermería) (Anexo I). Para la categorización de emociones primarias y secundarias y sentimientos se han seguido las orientaciones de Vigotsky (desde la perspectiva del aprendizaje sociocultural o constructivismo social donde se considera la incidencia de la interacción social en la construcción de las emociones y sentimientos) y Heller (considerando los principios de su teoría de los sentimientos) $(2,22)$.

Para determinar la unidad de observación y análisis de la población se han seguido las orientaciones de Martínez Salgado supeditando la investigación a su naturaleza, esencialmente cualitativa, y a la lógica que orienta y determina la potencia del muestreo mediante la selección intencional de los casos elegidos para que proporcionen la mayor riqueza de información posible (23). En consecuencia la muestra, coincidente con la población sujeto de estudio, ha estado compuesta por la totalidad de los 22 alumnos de Investigación Cualitativa asistentes al máster de enfermería, cuidados críticos y emergencias de la Universidad de Jaén (España) durante el curso académico 2015-16 que tienen experiencia clínica, ya sea como alumnos en prácticas o como profesionales. Asimismo, se elaboró un cuestionario integrado por estas categorías: emociones primarias, emociones secundarias y sentimientos (Anexo II). Este cuestionario se pasó a los alumnos tras explicarles las características de cada una de esas categorías y solicitarles que identificaran aquellas que consideraran como parte de su experiencia clínica de forma retrospectiva.
El análisis de los datos se realizó siguiendo las aportaciones de Siles y Solano mediante un proceso de reducción, organización en bloques temáticos y categorización de emociones primarias, secundarias y sentimientos en sus correspondientes subcategorías (5). Se siguieron las pautas del programa de análisis de datos cualitativos Atlas-ti y se establecieron grupos de familias de emociones y sentimientos vinculados mediante palabras clave compartidas y, asimismo, grupos de redes integrados por familias.

Tanto el taller de poesía como el cuestionario de emociones y sentimientos se realizaron durante los meses de marzo-abril de 2016. Para garantizar las cuestiones éticas, se revisaron por el comité de la Asociación Nacional de Historia y Antropología de los Cuidados y, asimismo, se les pasó un documento informativo requiriéndoles su firma, que explicitaba que todos los datos de carácter personal obtenidos en este estudio son confidenciales de acuerdo con lo estipulado en la Ley Orgánica de Protección de Datos de Carácter Personal 15/99.

\section{RESULTADOS Y SU DISCUSIÓN}

En este trabajo se confirma que el proceso de enfermería como método de trabajo que centra el cuidado en el paciente influye de forma determinante en el proceso de interacción entre alumno-paciente, dándole más profundidad e intensidad a las dimensiones cognitivas, sensomotoras y, sobre todo, afectivas $(14,17)$. Sin duda, el proceso de enfermería contribuye a la emergencia de las emociones en primer lugar y, posteriormente, a la construcción social de los sentimientos (12). Este proceso se produce mediante un mecanismo de "contagio emocional" en el que se distinguen dos facetas: emociones que se derivan de cuidados que resultan gratificantes tanto para el paciente como para el alumno (que son la mayoría: administrar la medicación, ayudar a una madre a colocarse el bebé recién nacido, etc.); emociones resultantes de cuidados que generan estrés para el alumno y/o el paciente (realizar una cura intrusiva, cuidados post-mortem, comunicación de malas noticias a familiares, etc.) y que pueden contribuir a generar un estado de "burnout"(24 - 26).

La más importante aportación de la poesía a la enfermería radica en la clarificación de su naturaleza transcendente mediante la revelación de su objeto sujeto: el ser humano, la persona como núcleo, como materia prima con la que trabaja la enfermera para "producir su obra". No hay ningún artista que trabaje con una materia prima tan frágil, delicada, compleja, individual, única e irrepetible..., he ahí su carácter transcendente. Sí, mediante la palabra lírica que destila los sentimientos transformados en versos, los profesionales de enfermería tienen una herramienta de deconstrucción eficaz y bella para constatar la auténtica naturaleza de la enfermería y reinterpretarla desde esquemas más fidedignos liberándose de la apabullante seducción del neopositivismo y la tecnología.

Respecto a las emociones y sentimientos identificados en el taller de poesía de los cuidados, se pueden distinguir dos tipos de emociones: las primarias y las secundarias (Gráfico I). Las emo- 
ciones primarias son las innatas y son las que se producen de forma irreflexiva expresándose de forma no verbal (Gráfico I):

a) Las emociones primarias de "alegría" ( gratificación, orgullo, realización, satisfacción, éxtasis, placer) son las que tienen un mayor porcentaje entre los alumnos (22,3\%). De todas ellas, la satisfacción ha sido la emoción primaria más experimentada.

b) Las emociones primarias relativas a la "tristeza" (aflicción, autocompasión, melancolía, desaliento, desesperanza, pena, duelo, soledad, depresión, nostalgia) ocupan el segundo lugar en cuanto a porcentaje $(18,27 \%)$. La pena ha sido la emoción primaria más experimentada en esta categoría.

c) Las emociones primarias integradas en el concepto "cólera" (molestia, enojo, atropello, mal genio, hostilidad) alcanzan el tercer lugar porcentualmente $(15,7 \%)$. La molestia ha sido la emoción primaria más sentida en esta categoría.

d) Las emociones primarias agrupadas en el concepto "miedo": ansiedad, nerviosismo, desconfianza, inquietud, terror, preocupación. El nerviosismo y la preocupación han sido las emociones primarias de mayor frecuencia en esta categoría.

Respecto a las emociones secundarias, que son resultado de la fusión o mezcla de las emociones primarias y pueden ser producto del aprendizaje a través de la experiencia, se obtuvieron los siguientes resultados (Gráfico II):

a) Las emociones secundarias integradas en el concepto "amor": aceptación, amabilidad, afabilidad, caridad, confian- za, dedicación, entrega, son las de mayor porcentaje (45\%). La emoción secundaria "dedicación" ha sido de mayor frecuencia en esta categoría.

b) Las emociones secundarias derivadas del concepto "sorpresa": asombro, estupefacción, maravilla, shock, han sido identificadas en el segundo lugar porcentualmente (37\%)." EI asombro" ha sido la emoción secundaria de mayor frecuencia en esta categoría.

c) Las emociones secundarias, identificadas con el concepto "vergüenza": arrepentimiento, humillación, mortificación, pena, remordimiento, culpa, (14\%). "La humillación" ha sido la emoción secundaria de mayor frecuencia en esta categoría

d) Las emociones secundarias identificadas en torno al concepto "aversión": repulsión, asco, desdén, desprecio, menosprecio, (4\%). En esta categoría las emociones secundarias de mayor frecuencia han sido "el desdén" y "el menosprecio".

En relación a los sentimientos, que se originan a través de la relación a través del tiempo entre sujeto y objeto conocido (Gráfico III), los Desagradables: timidez, vergüenza, ira, impaciencia, inseguridad, han alcanzado el mayor porcentaje (22, 29\%), siendo las de mayor frecuencia en esta categoría "la timidez" y "la inseguridad"; prácticamente igualado con los sentimientos. Los Morales: deber, cumplir, obligación (22, 28\%), siendo el sentimiento de mayor frecuencia en esta categoría "el deber"; y seguido de los sentimientos Altruistas: afecto, simpatía, apego $(19,25 \%)$, siendo" el apego" el de mayor frecuencia ; y en el último lugar los sentimientos estéticos: lo bello $(14,18 \%)$.

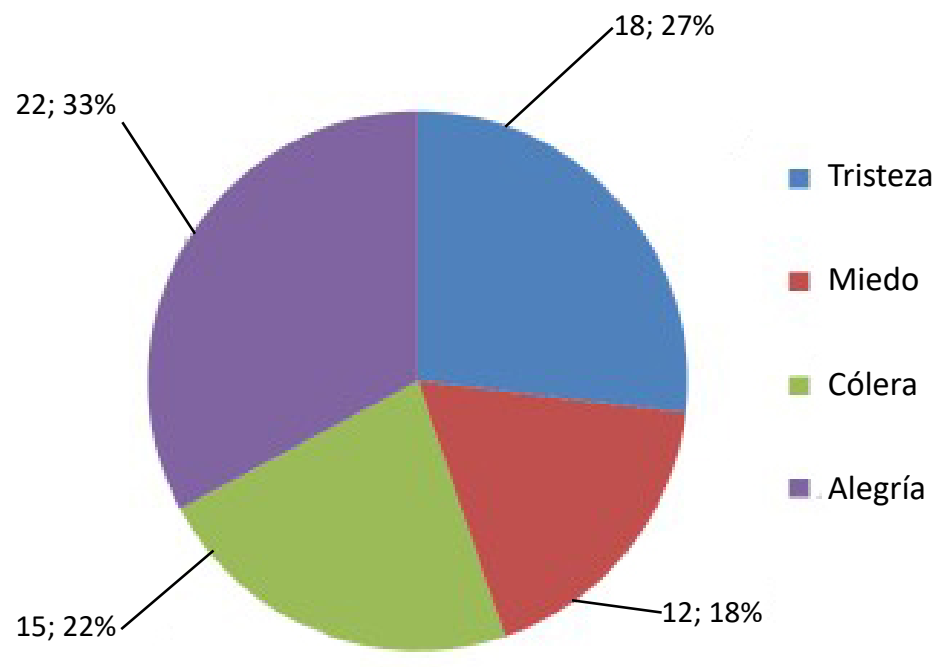

Gráfico I. Emociones primarias 


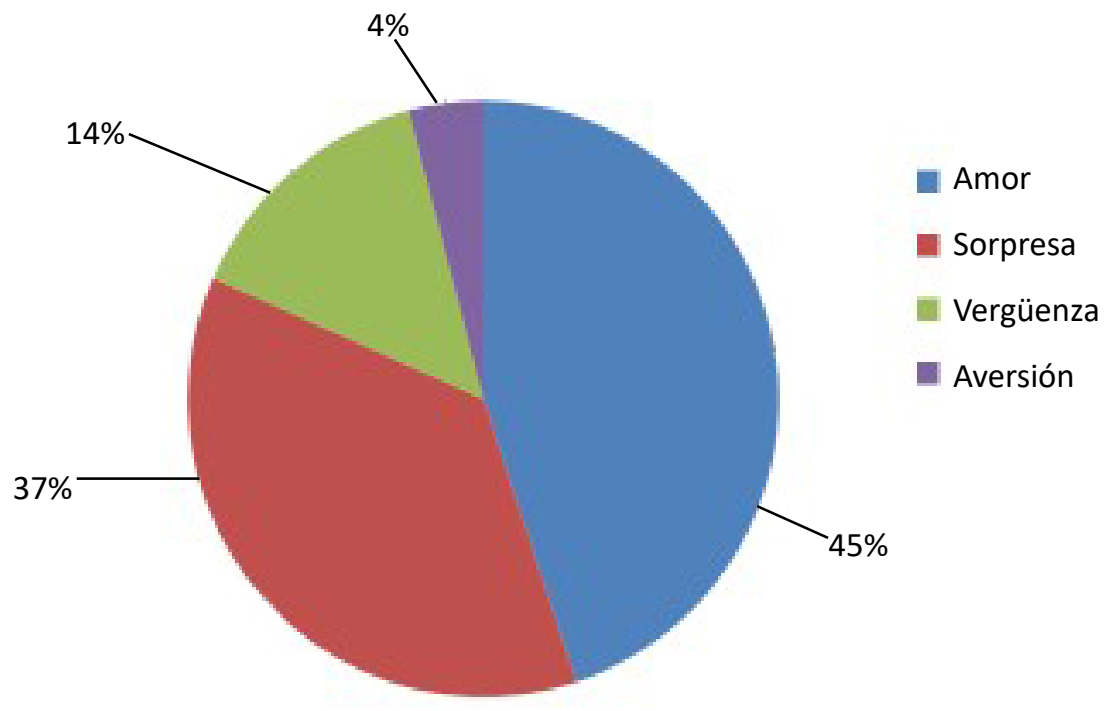

Gráfico II. Emociones secundarias.

Fuente: Elaboración propia partiendo de las respuestas al cuestionario de emociones primarias, secundarias y sentimientos (2017).

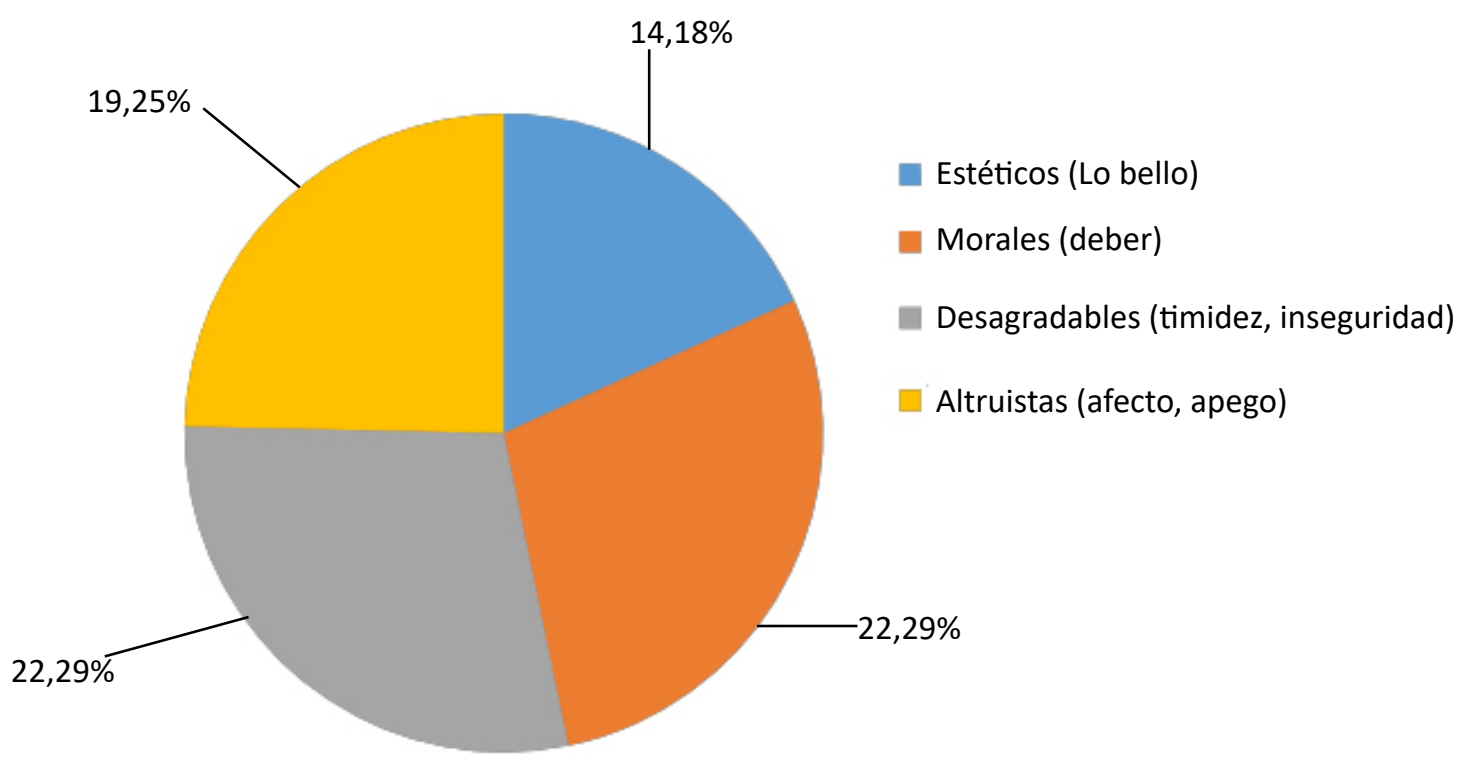

Gráfico III. Sentimientos

Fuente: Elaboración propia partiendo de las respuestas al cuestionario de emociones primarias, secundarias y sentimientos (2017). 


\section{LIMITACIONES DEL TRABAJO}

La identificación de las emociones, especialmente las primarias, ha tenido una dificultad especial para ser detectadas por los alumnos, dado su carácter espontáneo, sin tiempo a la reflexión y la rapidez de la respuesta conductual (aumento del ritmo cardiaco, sudoración, nervios, etc.). Las emociones primarias son las innatas, las que se producen de forma irreflexiva expresándose de forma no verbal (2). Por otro lado, aunque los sentimientos implican un estado afectivo plenamente consciente, con espacio a la reflexión y al aprendizaje pudiendo llegar a ser autónomos respecto a los estímulos ambientales, también resulta muy complejo identificarlos y valorar su incidencia en el rendimiento clínico. Se han explicado las características de los diferentes tipos de emociones y los sentimientos, pero aún así, ha resultado muy compleja la diferenciación de estas categorías por parte de los alumnos. Si a esto se añade el carácter retrospectivo del estudio (recordar a través del tiempo emociones y sentimientos vividos durante el trabajo clínico), todavía se aprecia más la dificultad de esta tarea. El taller de poesía y cuidados ha sido muy útil para facilitar la rememoración de los sentimientos y emociones vividos durante la práctica clínica.

\section{AGRADECIMIENTOS}

Este trabajo ha sido apoyado por el grupo de investigación de la Universidad de Alicante: Enfermería y Cultura de los Cuidados, y también del subgrupo de Antropología Educativa de los Cuidados en las Prácticas Clínicas integrado en el proyecto "Redes de Investigación en Docencia Universitaria" de la misma universidad.

\section{CONCLUSIONES}

Las emociones y los sentimientos potencian la actividad en el trabajo clínico si son positivos o la reprimen si son negativos.

- $\quad$ Se han identificado mayoritariamente y de forma retrospectiva las emociones y los sentimientos inspirados por poemas relacionados con la práctica clínica. -El principal método de trabajo de enfermería -el proceso de enfermería- tiene una gran incidencia en la construcción social de los sentimientos al potenciar la relación cuidador-cuidado.

- La poesía de los cuidados puede contribuir a la reflexión y gestión de emociones y sentimientos de alumnos durante trabajo clínico.

- Los sentimientos y emociones experimentados por los alumnos de enfermería durante sus trabajo clínico son tanto positivos como negativos, aunque los positivos prevalecen sobre los negativos mediante el desarrollo de procesos de adaptación y aprendizaje.

Es evidente la relación entre procesos históricos y socioculturales con la generación de emociones y sentimientos en un entorno tan especialmente proclive al impacto emocional como las prácticas clínicas.

- Resulta apropiado considerar la incidencia entre el proceso de construcción social y cultural de las emociones-sentimientos y la humanización de los cuidados.

Cómo citar este artículo: Siles-González, J; Solano- Ruiz, MC. Poesía y cuidados: un instrumento para la gestión de emociones y sentimientos en Enfermería. Enfermeria (montev.). [Internet]. 2017 Dic [citado xxxxx]; 6 (2) 32-38.

Disponible en: https://doi.org/10.22235/ech.v6i2.1463

\section{REFERENCIAS BIBLIOGRÁFICAS}

1. Darwin C. La expresión de las emociones en los hombres y los animales. Madrid: Alianza; 2009

2. Vigotsky L. Teoría de las emociones. Madrid: Akal; 2004

3. Sartre JP. Bosquejo de una teoría de las emociones. Madrid: Alianza, 2015

4. Betancourt L, Muñoz LA, Merighi MAB, Santos MF. El docente de enfermería en los campos de práctica clínica: un enfoque fenomenológico. Rev. Latino-Am. Enfermagem. 2011; 19(5): 9-13

5. Siles J, Solano MC. Antropología educativa de los cuidados. Una etnografía del aula y las prácticas clínicas. 2009; Alcoy: Marfil. Disponible en: https://rua.ua.es/dspace/bitstream/10045/20339/1/Antropologia\%20educativa.pdf

6. Escobar $\mathrm{MaO}$, González EM. Emociones y sentimientos durante las prácticas clínicas en el área de cuidado de los estudiantes del programa de enfermería de la universidad pontificia bolivariana.Cultura del Cuidado Enfermería. 2011; $1: 23$.

7. James N. Care = organisation + physical work + emotional labour. Sociology of Health \& Illness. 1992; 14(4): 488509. Disponible en: http://dx.doi.org/10.1111/1467-9566. ep10493127.

8. López SR. Implicación emocional en la práctica de la enfermería. Cultura de los Cuidados. 2000; 4(7/8): 172-180. Disponible en: https://rua.ua.es/dspace/bitstream/10045/5120/1/CC_07-08_20.pdf

9. Siles J, Solano MC. Cultural history and aesthetics of nursing care. Rev. Latino-Am. Enfermagem. 2011; , 19 (5): 1096-1105.

10. Siles J. El humanismo en enfermería a través de la sociopoética y la antropoética. Cultura de los Cuidados. 2014; 18, 38. Disponible en http://dx.doi.org/10.7184/ cuid.2014.38.01

11. Siles J, Solano MC. Sublimity and beauty: A view from nursing aesthetics. Nursing Ethics. 2016; 23(2): 154-166. Disponible en: http://dx.doi.org/10.1177/0969733014558966

12. Harre R. The Social Construction of Emotions. Oxford : Blackwell; 1986.

13. Small E. Valuing the emotional labour of nursing. Nursing times. 1996; 91 (26): 40-41. 
14. Smith P. The nursing process: raising the profile of emotional care in nursing training. Journal of advanced nursing. 1991; 16 (1): 74-81.

15. Smith R. The emotional labour of nursing. London: Macmillan; 1992.

16. Staden $\mathrm{H}$. Alertness to the needs of others: a study of the emotional labour of caring. Journal of advanced nursing. 1998; 27 (1): 147-56.

17. Waldow VR. Enseñanza de enfermería centrada en el cuidado. Aquichan. 2009; 9 (3): 246-56.

18. Birx E. The poetry of nursing. Clin Nurse Spec. 1994; 8(6):292-93.

19. Holmes V, Gregory D. Writing poetry: a way of knowing nursing. Journal of Advanced Nursing. 1998; 28: 11911194.

20. Silva O, Alves ED, Rodrigues MCS. Liricidad y toque de arte para la producción del conocimiento estético de enfermería -una reflexión poética inspirada en la Teoría de la complejidad. Cultura de los Cuidados. 2014;18(39): 14-29. Disponible en http://dx.doi.org/10.7184/cuid.2014.39.03

21. Siles J. Cultural Nursing Story: Epistemological and Methodological Reflection. Avances en Enfermería. 2010; 28 (1): 120-128.

22. Heller A. Teoría de los sentimientos. México: Ediciones Coyoacán; 2004.

23. Martínez-Salgado C. El muestreo en investigación cualitativa. Principios básicos y algunas controversias. Ciência \& Saúde Coletiva. 2012; 17(3):613-619.

24. Walster Hatfield E, Cacciopo P, \& Rapson, J. Emotional contagion. Cambridge: Cambridge University Press; 1994.

25. Brotheridge CM, Grandey AA. Emotional Labor and Burnout: Comparing Two Perspectives of "People Work". Journal of Vocational Behavior. 2002; 60: 17-39.

26. Bajo Gallego Y, González Hervías, R. La salud emocional y el desarrollo del bienestar enfermero. Metas Enferm. 2014; 17(10): 12-16 